\section{Aggressive Digital Papillary Adenocarcinoma}

\section{Pragya Attri and Prashanth Hari Dass*}

\author{
Lakes District Health Board, Rotorua, New \\ Zealand
}

\author{
* Corresponding author: \\ Prashanth Hari Dass
}

We report an interesting case of aggressive digital papillary adenocarcinoma (ADPCA), a rare eccrine neoplasm with high rates of recurrence and metastatic potential. Diagnosis and treatment is often delayed due to its unusual clinical presentation and it may be confused with other benign lesions. A man aged 71 was referred to the medical oncology clinic with CT scans showing recurrent oligometastatic ADPCA. Initial trauma more than a decade ago to the fourth digit lead to recurrent swelling necessitating a biopsy and subsequent amputation. Routine imaging surveillance then showed recurrence in the axillary lymph nodes for which the patient underwent an axillary node clearance. His most recent scan now shows a growing metastatic pulmonary nodule which is in consideration of resection. There is limited documentation on management of such cases. It is also important not to overlook the possibility of ADPCA when a patient presents with recurrent digital swellings or lesions.

Keywords: ADPCA; Eccrine neoplasm; CT scans
Lakes District Health Board, Rotorua, New Zealand.

ఏpranava108@hotmail.com

Tel: +64210490835

Citation: Attri P, Dass PH (2018) Aggressive Digital Papillary Adenocarcinoma. J Rare Disord Diagn Ther. Vol.4 No.3:14

Received: June 09, 2018; Accepted: July 03, 2018; Published: July 10, 2018

\section{Introduction}

Aggressive digital papillary adenocarcinoma (ADPCA) is a rare neoplasm of the sweat gland that was first described by Helwig in 1984 [1]. It is often misdiagnosed based on non-specific clinical and histological findings which can delay definitive surgical excision or amputation. ADPCA typically presents as a single nodular lesion on a digit, enlarging over months to years, predominantly in males aged between fifty and seventy [2]. Studies suggest that the histological picture does not necessarily correlate with predictability of recurrence or metastases [3]. Here we highlight a case of ADPCA that is parallel to previously documented cases [4-13].

\section{Case Report}

A 56 year old male non-smoker with no significant past medical history developed local swelling to the fourth digit of his right hand following a crush injury, which was then treated with minor orthopaedic debridement. Swelling which initially resolved later recurred eight years later. The patient sought medical attention and had an excision at the distal phalanx. Biopsy from excision showed an initial diagnosis of acrospiroma, however swelling recurred along with pain and discolouration and therefore amputation of the distal phalanx was required. Repeat biopsy showed appearances consistent with a diagnosis of aggressive digital papillary adenocarcinoma. However this was not noted until another five years later, when the swelling and pain recurred. Magnetic resonance imaging confirmed a $12 \times 8 \times 10 \mathrm{~mm}$ soft tissue lesion involving bone (Figure 1) and further amputation of the whole fourth digit was required. On this occasion, the biopsy reported a tumour nodule which was well delineated but unencapsulated, with no lymphovascular space or perineural invasion; soft tissue resection had clear margins.

In view of the nature of ADPCA, the patient was put under regular surveillance with computed tomography (CT) imaging of the chest, abdomen and pelvis. A few months after complete digit amputation, a CT showed a $25 \mathrm{~mm}$ heterogeneously enhancing lymph node in the right axilla (Figure 2). He underwent total axillary clearance, in which one of seventeen lymph nodes was proven positive. The patient had a consultation with radiation oncologists to discuss the merits of adjuvant radiotherapy to reduce the risk of regional recurrence. The risk was deemed to be between 10 and $15 \%$ and would be further reduced to $5 \%$ if radiotherapy was administered to the surgical field and supraclavicular fossa. Upon consideration of potential side effects however, the patient declined this treatment. Two years later further CT imaging showed a right upper lobe pulmonary nodule, most typical in appearance for a pulmonary metastasis. This had increased from $3 \mathrm{~mm}$ to $9 \mathrm{~mm}$ in maximum axial dimension over 


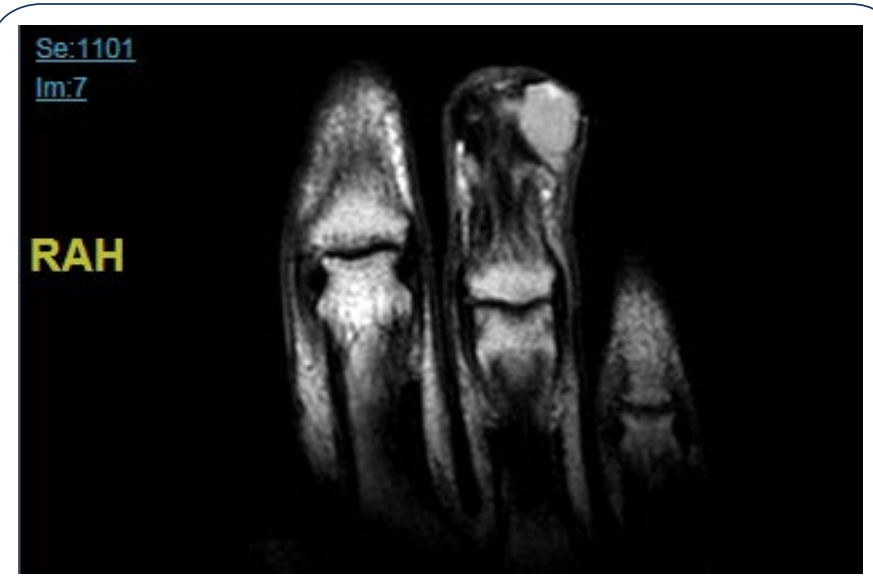

Figure 1 Magnetic Resonance Imaging of right fourth digit showing soft tissue lesion invading into bone cortex.

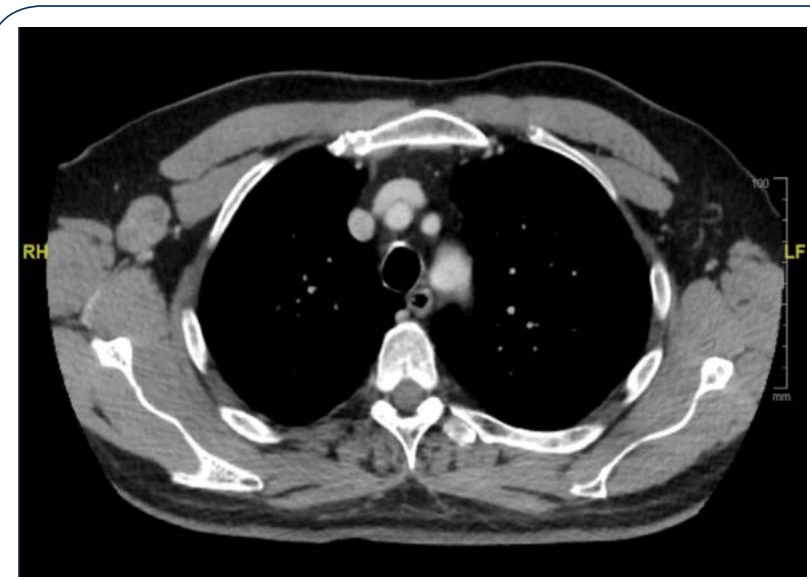

Figure 2 Computed Tomography scan showing a $25 \mathrm{~mm}$ right axillary node.

6 months (Figure 3) and then to $12 \mathrm{~mm}$ as suggested by PET-CT two months later. A second nodule in the left lower lobe was also seen and deemed to be long-standing and favoured to be benign or inflammatory. There were no other distant sites of metastases. With this gradual progression of ADPCA with oligometastases and limited data available regarding suitable radiotherapy, chemotherapy or immunotherapy, systemic treatment was not given. The patient has remained asymptomatic, with no cough or dyspnoea. His Eastern Cooperative Oncology Group performance status is 0 and clinical examination was unremarkable. Laboratory investigations have been unremarkable. He is currently waiting for an opinion from cardiothoracic surgeons regarding possible lung wedge resection of the single pulmonary lesion.

\section{Discussion}

Aggressive digital papillary carcinoma was first described by Helwig in 1984 [1] and later by Kao G. F. in 1987. Kao studied 57 cases and divided neoplasms into adenoma (ADPA) and adenocarcinoma (ADPCA), with ADPCA being distinguished by poor glandular differentiation, necrosis, cellular atypia and pleomorphism, and invasion into surrounding tissue [4]. This was considered important to guide clinical therapy [4] but subsequent studies strongly suggested that all tumours were aggressive in nature and the histological picture did not correlate with predictability of rate of recurrence and metastases [3]. Regardless of malignant potential, tumour recurrence rate was reported to be $50 \%$ and tumour metastasis was $14 \%$ with or without recurrence [5]. Based on their high potential to be aggressive, in 2006 the World Health Organization classified all digital eccrine tumours as digital papillary carcinoma, with no benign counterpart. ADPCA tumours characteristically present as slow growing, solitary non-tender masses that arise from the fingers and toes, with the distal part of the finger or thumb being most frequently documented [5]. They occur predominantly in men at a 9:1 male to female ratio, most commonly in the $5^{\text {th }}$ and $6^{\text {th }}$ decade of life [2]. Histological features typically include a solid and cystic growth pattern with papillae formation (Figure 4), with fused back-to-back tubules lined by cuboidal to low epithelial cells, surrounded by an outer myoepithelial layer. Tumours may or may not be well circumscribed $[7,8]$. In our case, the first excision demonstrated nests and cords of cells, some basaloid and some larger paler cells surrounded by hyalinising dense fibrous stroma. This is in keeping with a solid and cystic hidradenoma (acrospiroma) which should be considered in the differential

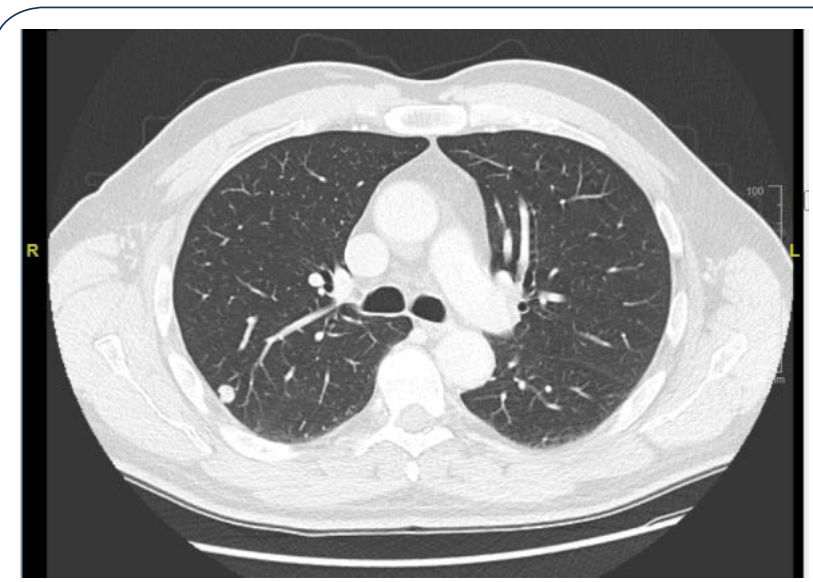

Figure 3 Computed Tomography scan showing a right pulmonary nodule.

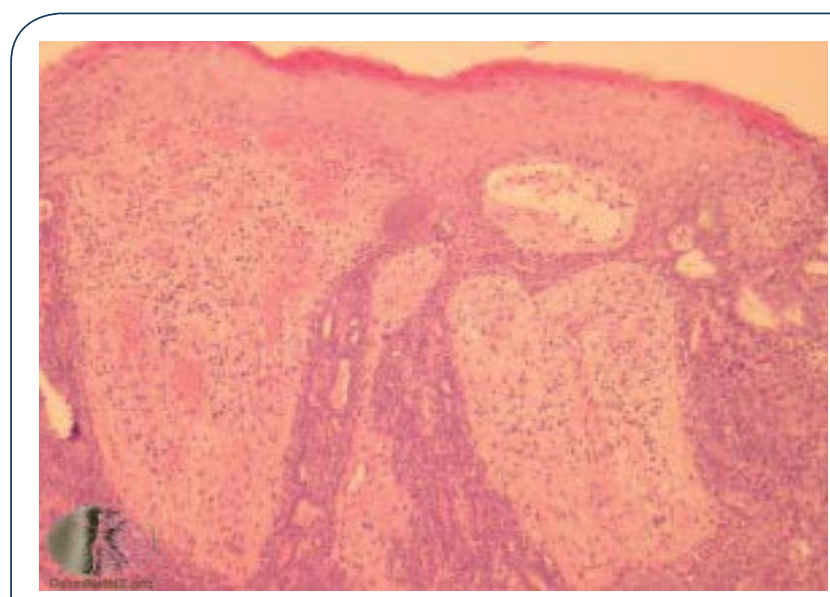

Figure 4 Typical histological picture seen in ADPCA (does not belong to this case), illustrating a predominantly solid and ductal morphology with focal areas of papillary formation. The cells are basaloid, show enlarged atypical nuclei, large nucleoli and increased mitoses. 
diagnosis. In contrast, hidradenoma predominantly occurs in the head and neck region, with a preponderance to elderly women [9]. Subsequent biopsies, however, showed a more destructive growth pattern with obvious permeation of phalangeal bone, presence of basaloid cells with areas of squamous cell differentiation, areas of papillary type growth pattern and focal duct formation, all consistent with ADPCA. Immunohistochemistry from a retrospective case series of seventeen evaluable patients showed positivity for p63, keratin 7, keratin 77 (eccrine ductspecific), PHLDA1, and epithelial membrane antigen in most cases, and for carcinoembryonic antigen, smooth muscle actin, S100 protein, estrogen, progesterone, and androgen receptors in $50 \%$. Mean Ki67 proliferation index was 15\% [10]. Diagnosis and treatment of ADPCA is often delayed due to misdiagnosis; it can easily be confused with soft tissue infections, ganglion cysts, calluses, pyogenic or foreign body granulomas, hemangiomas, osteomyelitis or gout [8]. Other tumour types should also be ruled out, including adnexal tumours, squamous cell carcinomas and giant cell tumours. It is important to also consider metastatic carcinoma of the breast, lung and thyroid [12] which can resemble ADPCA by similar histopathological findings including hyperchromatic nuclei, brisk mitotic activity, tumour infiltration into the surrounding stroma and necrosis [13]. Initial and definitive treatment for ADPCA is surgery; wide excision and partial digital amputation significantly reduces the chance of metastasis and recurrence [3]. Amputation or re-excision reduces recurrence rates from $88 \%$ to $25 \%$ [14]. Lymph node metastasis has previously been reported in approximately $9-12 \%$ of cases $[3,7]$, therefore sentinel lymph node mapping has also been recommended. Distant metastases are reported to occur in 14-

\section{References}

1 Helwig EB (1984) Eccrine acrospiroma. J Cutan Pathol 11: 415-420.

2 Nash M, Burn J, Chan S (2016) An unexpected digital papillary adenocarcinoma of the thumb. Case Reports Plast Surg Hand Surg 3: 77-78.

3 Suchak R, Wang W, Prieto VG, Ivan D, Lazar AJ, et al. (2012) Cutaneous Digital Papillary Adenocarcinoma. Am J Surg Pathol 36: 1883-1891.

4 Kao GF, Helwig EB, Graham JH (1987) Aggressive digital papillary adenoma and adenocarcinoma. A clinicopathological study of 57 patients, with histochemical, immunopathological, and ultrastructural observations. J Cutan Pathol 14: 129-146.

5 Lim S, Cho I, Lee MJ (2014) Digital Papillary Carcinoma. Korean J Pathol 48: 438-441.

6 Bryant JR, Gardner P, Yousif M, Pui JC, Hajjar RT, et al. (2017) Aggressive Digital Papillary Adenocarcinoma of the Hand Presenting as a Felon. Case Rep Orthop 2017: 1-5.

7 Duke WH, Sherrod TT, Lupton GP (2000) Aggressive Digital Papillary Adenocarcinoma. Am J Surg Pathol 24: 775-784.

8 Kobayashi T, Hiura A, Oishi K, Maeda S, Ohara K, et al. (2016)
$22 \%[3,7]$ with the most frequent site being the lung parenchyma. Other sites that have been documented include brain, skin, bone, small bowel and kidney $[4,7]$. Haematogenous spread can occur without lymph node involvement [8] therefore starting regular surveillance by imaging early is warranted to monitor the natural course of the disease. There are very few documented cases, in particular in patients with pulmonary metastases, where adjuvant systemic treatment in the form of chemotherapy has been trialled. Examples of drugs used include carboplatin and paclitaxel, docetaxel, gemcitabine, 5-fluoruracil and mitomycin. These have not shown to have any obvious progression free survival or overall survival benefit $[6,8,15]$. Surgical excision for oligometastases may be warranted as in the case of our patient however there is limited evidence to support this. To summarise, ADPCAs are rare eccrine neoplasms primarily found in males with a predilection for the digits of the hand. They have a tendency to be slow growing with non-specific clinical and histopathological features, and clinicians should consider ADPCA in their differential diagnosis of hand and foot which may mimic benign lesions. These tumours have a high incidence of recurrence after wide excision and significant metastatic potential, most frequently spreading to regional lymph nodes and lungs. Patients should be monitored long-term with regular clinical examination and CT imaging for consideration of metastectomy.

\section{Acknowledgement}

The authors would like to acknowledge the patient involved in this case for providing consent to write this report, as well as the orthopaedic team, pathologists and radiation oncologists who were involved in his care.

Aggressive Digital Papillary Adenocarcinoma with Multiple Organ Metastases. Am J Dermatopathol 38: 910-914.

9 Al-Rohil (2017) Evening Specialty Conference Dermatopathology. https://handouts.uscap.org/AN2017/2017_EC06_Rohil_0401_post.pdf

10 Weingertner N, Gressel A, Battistella M, Cribier B (2017) Aggressive digital papillary adenocarcinoma: A clinicopathological study of 19 cases. J Am Acad Dermatol 77: 549-558.

11 Emanuel P (2014) Aggressive digital papillary adenocarcinoma pathology. https://www.dermnetnz.org/topics/aggressive-digitalpapillary-adenocarcinoma-pathology/

12 Laun J, Gopman J, Barnes CW, Segars, KA, Elston JB, et al. (2015) Aggressive Digital Papillary Adenocarcinoma. Eplasty 15: 60.

13 Geethamani V, Shetty A, Chiwdappa V (2014) Aggressive Digital Papillary Adenocarcinoma-A rare Entity Posing a Diagnostic Challenge. Malays J Med Sci 21: 54-56.

14 http://meeting.handsurgery.org/files/2015/Presentations/P69.pdf

15 Jones JA, Patel VB, Goldsmith B, Teitelbaum U, Plastaras JP, et al (2013) Diffusely Metastatic Digital Papillary Adenocarcinoma 11 Years After Initial Presentation Treated With Palliative Chemotherapy and Radiotherapy. J Clin Oncol 31: e386-e389. 